\title{
Long-length and thermally stable high-finesse Fabry-Perot interferometers made of hollow core optical fiber
}

\author{
Meng Ding, Matěj Komanec, Dmytro Suslov, Daniel Dousek, Stanislav Zvánovec, Eric R. Numkam \\ Fokoua, Thomas D. Bradley, Francesco Poletti, David J. Richardson, Fellow, IEEE, Fellow OSA, and \\ Radan Slavík, Senior Member, IEEE, Fellow OSA
}

\begin{abstract}
We made and characterized two Fabry-Perot interferometer samples made of the latest-generation hollow core fiber with sub-1-dB/km loss. Thanks to this low transmission loss, we achieved a finesse of over 140 and 120, for interferometer lengths of 5 and $23 \mathrm{~m}$, respectively. This resulted in transmission peaks as narrow as $47 \mathrm{kHz}$. Our all-fiber Fabry-Perot interferometers have standard single-mode fiber pigtails (for easy integration in conventional fiber optic systems) and employ fiber mode field adapters to enable low-loss coupling between the pigtails and the low-loss hollow core fiber. The high-reflectivity mirrors $(>98 \%)$ were deposited directly on the fiber mode field adapters, which were glued to the hollow core fiber, resulting in permanently-aligned Fabry-Perot interferometers. We also measured how the position of the transmission peaks change with temperature (an important performance metrics for most applications, e.g., when used as a narrow-band band-pass filter) and found that it changed 14.5 times less in our Fabry-Perot interferometer relative to a similar device made of standard single mode fiber.
\end{abstract}

Index Terms-Optical fiber devices, Optical fiber applications, Fabry-Perot

\section{INTRODUCTION}

$\mathrm{F}$ ABRY-Perot interferometers (FPs) have many unique properties including high wavelength selectivity, high extinction ratios, the capability to enhance light intensity, etc. Their applications include, amongst others: lasers [1]-[3], metrology [4], [5] and sensing [6]-[8]. The full width at half maximum, FWHM of the transmission peaks is the key parameter of a FP, as a narrower peak provide better capability to discriminate frequency [6], of relevance, e.g., in reference cavities [2] or a ultra-high resolution sensors [5]. Another important parameter is the free spectral range (FSR), which is the spacing between neighboring transmission peaks and which is inversely proportional to the FPs optical length $n L$, where $n$ is the refractive index and $L$ is the length. The finesse $(F)$ is then defined as the ratio FSR/FWHM. A small FWHM can be

Manuscirpt received December $\mathrm{xx}$, $\mathrm{xxxx}$; revised February $\mathrm{xx}, \mathrm{xxxx}$; accepted xx, xxxx. This work was supported by EPSRC project "Airguide Photonics", under grant EP/P030181/1 and Czech Technical University in Prague (SGS SGS17/182/OHK3/3T/13). Meng Ding is supported by CSC scholarship. Francesco Poletti gratefully acknowledges EU ERC (grant 682724); Radan Slavík was supported by RAEng Fellowship. achieved with a high finesse or small FSR (or both). In freespace FPs (made, e.g., by drilling a hole in a low-expansion glass rod and then attaching high reflectivity mirrors at both ends [4]), $L$ is usually limited by practical constraints to less than $50 \mathrm{~cm}$, limiting the achievable FSR. Thus, if a small FWHM is desired, ultra-high finesse (sometimes $>10^{5}$ ) is needed, making such FPs highly sensitive to alignment. Thus, an alternative implementation using single-mode fiber FPs (SMF-FPs) has been widely investigated. SMF-FPs can have long lengths (e.g., 100's of meters) and can be very compact and lightweight. Although the finesse of SMF-FPs [7], [8] does not reach that achievable in free-space FPs (due to the fiber transmission loss), their small FSR enables narrow transmission peaks similar to those of short, high-finesse free-space FPs. Unfortunately, SMF-FPs have several drawbacks that make free-space FPs the preferred approach for many applications, despite free-space FPs larger size and the alignment challenges. The two primary drawbacks are the large sensitivity to temperature variations [8] and unwanted nonlinear effects like stimulated Brillion scattering (SBS) [9], especially in highfinesse FPs where the intra-cavity power is strongly enhanced by the resonant effect. Both of these parasitic effects are mainly due to the interaction of light with the silica glass material in SMFs. For example, the thermal sensitivity of the FSR (determined by the temperature dependence of the optical length $n L$ of the SMF) is dominated (95\%) by thermallyinduced changes in the $\mathrm{n}$ of silica glass (thermo-optic effect) with the thermally-induced fiber length (thermal expansion effect) change providing the other 5\% [10].

Hollow core fibers (HCFs) in which the glass-light interaction is very weak should address shortcomings discussed above. Firstly, the thermo-optic effect is practically eliminated, making HCF 20 times less thermally sensitive than SMF of equal $L[10]$. When considering equal optical length $n L$, this advantage is reduced (as $n \sim 1$ in HCF compared to $n \sim 1.45$ in SMF) to about 14 times [11], [12]. Nonlinearities are also substantially weaker in HCF as compared to $\mathrm{SMF}$, enabling several orders of magnitude higher powers to be

Meng Ding, Eric R. Numkam Fokoua, Thomas D. Bradley, Francesco Poletti, David J. Richardson and Radan Slavík are with the Optoelectronic Research Centre, University of Southampton, SO17 1BJ, UK (R.Slavik@ soton.ac.uk)

Matěj Komanec, Dmytro Suslov, Daniel Dousek, and Stanislav Zvánovec are with the Faculty of Electrical Engineering, Czech Technical University in Prague, 16627 Prague, Czech Republic. 
launched into HCF-FPs as compared to SMF-FPs [1].

Several recent works have reported high-finesse HCF-FPs. The cavity mirrors were formed using photonic crystal slabs [13] or high-reflectivity dielectric coatings [14]. However, all these reports dealt with $\mathrm{cm}$-long fiber lengths limiting their use for applications requiring narrow FWHM transmission peaks. Generally, they used 7-cell photonic bandgap HCF designs, which offer good coupling into SMF, but whose high loss (>10 $\mathrm{dB} / \mathrm{km}$ [15]) does not allow the construction of high-finesse long-length HCF-FPs (e.g., for 20-m long FP made with 10$\mathrm{dB} / \mathrm{km}$ fiber, the fiber-loss-limited maximum achievable finesse is 68). Furthermore, the reported HCF-FPs were not designed for long-term operation since their ends were not sealed, enabling air-born pollution like water vapor and dust to enter the hollow core, possibly causing long-term HCF-FP performance degradation.

Concerning ongoing efforts to reduce HCF loss, antiresonant type HCFs have recently witnessed tremendous progress [16][18]. The most recent results obtained with the Nested Antiresonant Node-less Fiber (NANF) geometry have broken the $1-\mathrm{dB} / \mathrm{km}$ barrier $(0.65 \mathrm{~dB} / \mathrm{km}$ across a bandwidth of $120 \mathrm{~nm}$ [18]). This has opened up a host of new application opportunities, including the possibility of making long length high-finesse HCF-FPs.

In this paper, we demonstrate and characterize two HCF-FPs with lengths of 5 and $23 \mathrm{~m}$, with finesse > 120 over a wavelength range from $1530-1565 \mathrm{~nm}$, made using NANF with sub-1 dB/km loss and pigtailed with SMF. These HCF-FPs are two orders of magnitude longer than previously-demonstrated. We compare their thermal stability with that of an SMF-FP, demonstrating more than an order of magnitude better performance. Besides improving the stability of fiber-based FPs, this also represents the first demonstration of such low thermal sensitivity in antiresonant HCFs; to date this has only been previously demonstrated for photonic bandgap type HCFs.

\section{FABRICATION OF HCF-FPS}

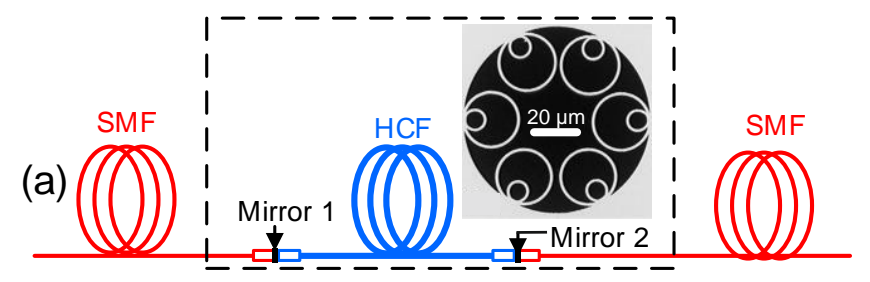

(b)

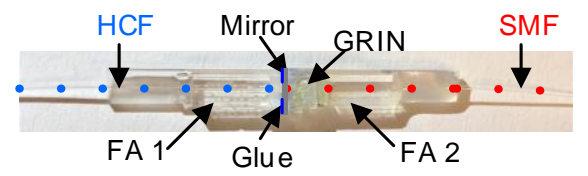

Fig. 1. (a) Configuration of the pigtailed HCF-FP with a cross-sectional scanning electron microscope (SEM) image of the HCF used. (b) Photograph of the fully assembled SMF-GRIN-Mirror-HCF interconnection. FA: fiber array, GRIN: Graded-index fiber based mode field adapter.

The schematic of the pigtailed HCF-FP is shown in Fig. 1. The HCF used is a NANF similar to the one reported in [18] with a loss of $0.9 \mathrm{~dB} / \mathrm{km}$, operating in the $1550-\mathrm{nm}$ wavelength region. The HCF-FP is pigtailed with SMF. Because of the mode mismatch between the fundamental mode of the HCF used $(21 \mu \mathrm{m})$ and standard SMF $(10 \mu \mathrm{m})$, a suitable piece of GRIN fiber was inserted in between the two to enable mode field adaptation [19]. The GRIN fiber was first fusion spliced to the SMF. Before connecting it to the HCF, a FP mirror was deposited on its end-face. We used a 13-layer $\mathrm{Ta} 2 \mathrm{O} 5 / \mathrm{SiO} 2$ dielectric coating-based mirror with a reflectivity of $>98 \%$ (from 1500-1570 nm). We did not use fusion splicing to interconnect the mirrors to the $\mathrm{HCF}$, as this would destroy the mirror and could cause deformation of the HCF microstructure. We also did not use mechanical splice as shown in [14], since this approach does not allow for active alignment of the angle between the NANF and the mirror, imposing inherent limitation to reproducibility as well as the maximum achievable finesse. Instead, we used a modified fiber-array technique (used industrially for fiber pigtailing of planar lightwave circuits, PLCs) which we have refined for low-loss interconnection $(<0.3 \mathrm{~dB} /$ interconnection) between HCFs and SMF [19]. Here, the SMF spliced to the GRIN is first glued into a V-groove array, polished to the desired GRIN fiber length, and afterwards, a coating is deposited on the end facet (the mirror in the work presented here). Then the carefully cleaved NANF is inserted into another fiber array $\mathrm{V}$-groove and glued in to that. The two fiber arrays are actively aligned (in $\mathrm{x}, \mathrm{y}, \mathrm{z}$, pitch, and yaw) before gluing the fiber arrays together (to obtain a permanent and sealed interconnection, which does not allow any air-born pollutants to enter the hollow core cavity, and thus ensures no degradation in the long-term performance). A Photograph of a typical glued interconnection is shown in Fig. $1 \mathrm{~b}$.

\section{Broadband}

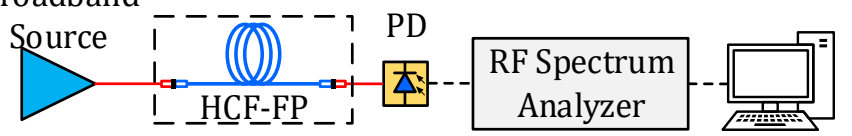

Fig. 2. Set-up for characterization of FPs in the radio-frequency (RF) domain. The broadband source is formed by two cascaded Erbium-doped fiber amplifiers (EDFAs) with a tunable bandpass filter inserted in between them. PD: photodetector.

Since we target long length high-finesse FPs which have very narrow transmission peaks, optical scanning method used, e.g., in [13] is not ideal for active cavity alignment prior to gluing. This is because too many parameters are changing during the alignment (FP peaks spectral position, insertion loss, polarization, etc.), making alignment tedious and slowly converging. We found that in practice, the following two methods (for coarse and fine alignments) allowed us to align the HCF-FP cavity relatively quickly. For the coarse alignment, we optimized transmitted optical power of the HCF-FP using a 10$\mathrm{nm}$ wide broadband incoherent light source and a photodetector. Once maximum power has been reached, we analyzed the photodetected signal with a radio-frequency (RF) spectrum analyzer observing RF beats (discussed in detail later) [20], Fig.2. In the coarse method, the transmitted power is insensitive to HCF-FP peaks spectral position and polarization state inside the FP. By maximizing the transmitted power, coupling loss between the HCF and the mirrors (i.e., how much light at the HCF output is coupled back into it) is minimized, which in practice allows for almost-optimum HCF-FP cavity alignment. The RF beat analysis technique allowed us to 
measure FP finesse directly (as we show below) enabling us to fine tune the FP. The signal shown on the RF spectrum analyzer is proportional to the convolution of the FP's optical coefficient with its conjugate:

$$
P(f) \propto \frac{1}{1+\left(\frac{2 F_{e}}{\pi}\right)^{2} \sin ^{2}\left(\frac{\pi f}{F S R}\right)} .
$$

Here, $f$ is the signal frequency shown on the RF spectrum analyzer and $F_{e}$ is:

$$
F_{e}=\frac{\pi R}{1-R^{2}} \approx \frac{\pi \sqrt{R}}{2(1-R)}=\frac{F}{2} .
$$

where $R=\sqrt{R_{1}} \sqrt{R_{2}} \alpha$ is the effective reflectivity of the interferometer with $R_{1,2}$ being the reflectivities of the mirrors, and $\alpha$ the single-trip loss including the coupling loss between the HCF and the mirrors (i.e., how much light at the HCF output is coupled back into it) and fiber transmission loss. Although Eq. (1) is identical in form to the expression for the FP's optical transmission transfer function [13] it is not the same since $F_{e}$ is different to the finesse $F$. For a high-finesse FP in which $R \sim 1$, we have derived that $F \sim 2 F_{e}$ based on the first order Taylor series approximation. Thus, the FP's RF spectrum has twice as large peaks compared to the optical spectrum. In our experiment, data from the RF spectrum analyzer were acquired with a computer, where they were fitted with Eq. (1) in LABVIEW, enabling the FSR and finesse to be output in realtime, allowing for active FP alignment.

We made two HCF-FP samples using 5 and $23 \mathrm{~m}$ long HCFs. When aligned (prior to gluing), their Finesses were 195 and 160 , respectively. After gluing using a UV-curable glue, we witnessed a slight finesse reduction to 153 and 133, respectively. Although we believe this may be improved by further refining the gluing process, the high-finesse (>120) FPs obtained are robust and have not degraded with time (we have not seen any degradation in four months since gluing them).

The 23-m HCF-FP had a smaller finesse both before and after the gluing as compared to the 5-m device. By considering the HCF loss of $0.9 \mathrm{~dB} / \mathrm{km}$ and mirror reflectivity of $98.6 \%$, analysis using Eqs. $(1,2)$ suggests that it is due to the HCF's transmission loss.

\section{Characterization}

Following the fabrication, we characterized the FPs using two techniques - besides the convenient, fast, and high-dynamic range RF technique we have described; we also measured it directly in the optical domain. We measured all of the important parameters (insertion loss, birefringence, and dependence on the wavelength). Finally, we measured the FPs' thermal sensitivity.

\section{A. Characterization in the RF domain}

The FP's transmission spectra including information about FSR and finesse were measured in RF domain using the method described previously. Normalized RF power spectra showing 5 peaks around $500 \mathrm{MHz}$ and normalized RF power spectra showing one peak with fitted curves for the fabricated HCF-FPs are shown in Fig. 3. The broadband source used for this measurement had a $1 \mathrm{~nm}$ optical bandwidth (this narrow bandwidth allowed us to perform spectrally-dependent characterization shown later) with a central wavelength of 1550 $\mathrm{nm}$ (given by the optical bandpass filter used in the source setup, Fig. 2). The small spikes at bottom of the RF power spectra are artefacts originating from noise in the EDFA electronics. We saw them in all our experiments. When fitting the experimental data with Eq. (1), we used only data above $-10 \mathrm{~dB}$ to avoid any contribution from the measurement noise. Despite this, we achieved a very good fit across the entire fitting range. The FSR and $F$ were $28.1 \mathrm{MHz}$ and 153 for the $5 \mathrm{~m} \mathrm{HCF-FP}$ and $6.5 \mathrm{MHz}$ and 133 for the $23 \mathrm{~m} \mathrm{HCF}-\mathrm{FP}$.
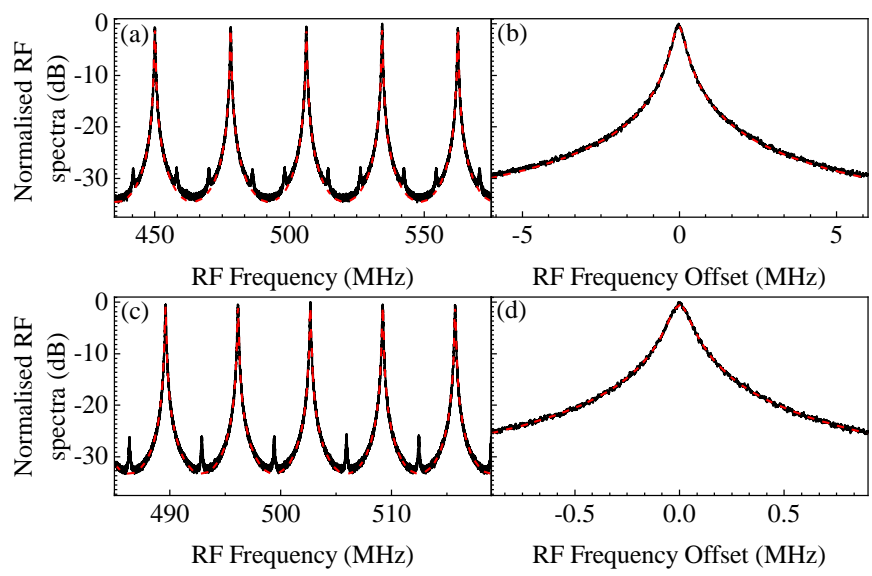

Fig. 3. Measured results for $5 \mathrm{~m}-\mathrm{HCF}-\mathrm{FP}(\mathrm{a}, \mathrm{b})$ and $23 \mathrm{~m} \operatorname{HCF}-\mathrm{FP}(\mathrm{c}, \mathrm{d})$. Normalized RF power spectra around $500 \mathrm{MHz}(\mathrm{a}, \mathrm{c})$ and their details around one peak (b, d). Solid black: measured, red dashed: fitted with Eq. (1).

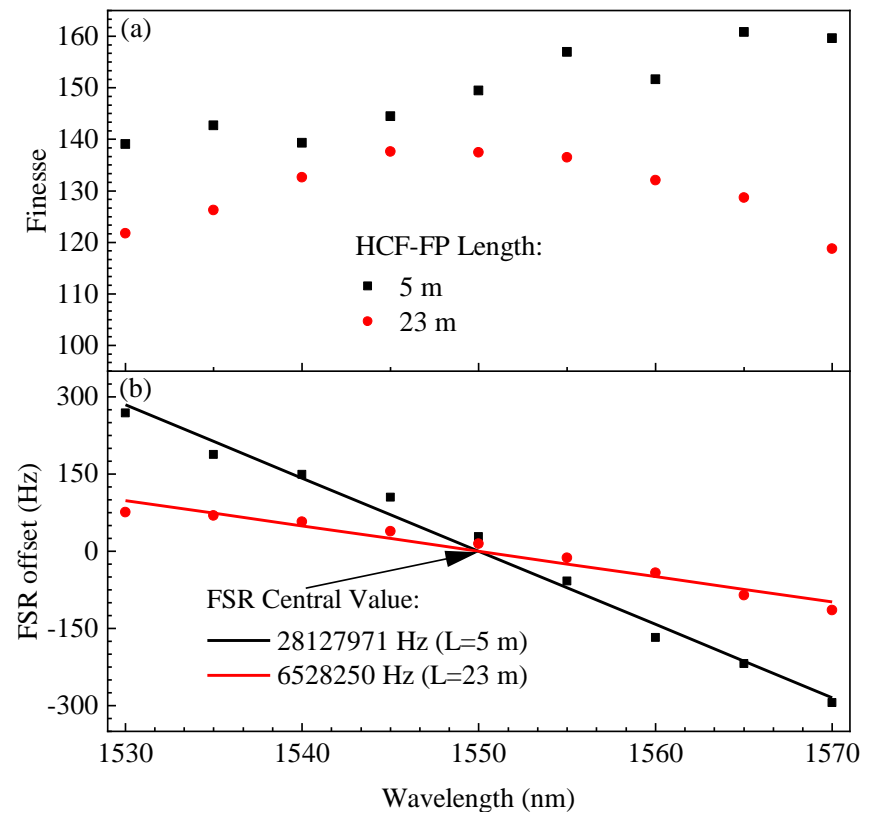

Fig. 4. (a) Finesse and (b) FSR offset of the two HCF-FP samples measured with the RF method every $5 \mathrm{~nm}$ across the $\mathrm{C}$ band.

Subsequently, we measured the RF spectrum of the HCF-FPs over the entire $\mathrm{C}$ band (by tuning the wavelength of the 1-nm bandwidth tunable bandpass filter, Fig. 2). The results of both FPs are displayed in Fig. 4(a) (finesse) and Fig. 4(b) (FSR). The finesse values range from 140 to 160 for the 5-m HCF-FP and 
from 120 to 138 for the 23-m HCF-FP. Considering this measurement data and their variation across the $\mathrm{C}$-band, the effective reflectivity (reflectivity that includes the coupling loss of light from the $\mathrm{HCF}$ to the mirror and back and $\mathrm{HCF}$ transmission loss) was calculated to be between $97.8 \%$ and 98.1\% (5-m HCF-FP) and $97.4 \%$ to $97.75 \%$ (23 m HCF-FP). The FSRs, Fig. 4(b) slightly decrease with wavelength (by 150 $\mathrm{Hz}$ for 5-m HCF-FP and $600 \mathrm{~Hz}$ for 23-m HCF-FP over the entire C-band). This is due to the HCF chromatic dispersion, which we confirmed by fitting the data in Fig. 4(b) and calculating HCF chromatic dispersion from them, obtaining value of $2 \mathrm{ps} / \mathrm{nm} / \mathrm{km}$. This is consistent with value expected for our NANF-type HCF [18]. It is worth mentioning that this value of chromatic dispersion is 8 times lower than SMF and thus our HCF-FP is expected to have 8 times smaller variation of its FSR as compared to SMF-FP.

\section{B. Characterization in the optical domain}

Further FP parameters such as insertion loss (FP transmission loss at resonance) and polarization dependence are less straightforward to measure using the RF analysis technique. Thus, we implemented a characterization technique operating directly in the optical domain, Fig. 5.

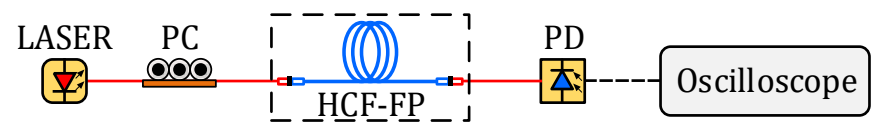

Fig. 5. Set-up to characterize the HCF-FPs in the optical domain. PC: polarization controller; PD: photodetector.

We scanned the input light frequency of a narrow-linewidth $(<10$ $\mathrm{kHz}$ ) fiber laser (NP Photonics, $1556 \mathrm{~nm}$ ) by applying a sawtooth waveform on its RF port. The output was photodetected and visualized on an oscilloscope. The laser RF port response was calibrated to obtain the spectral characteristics of the FP transfer.
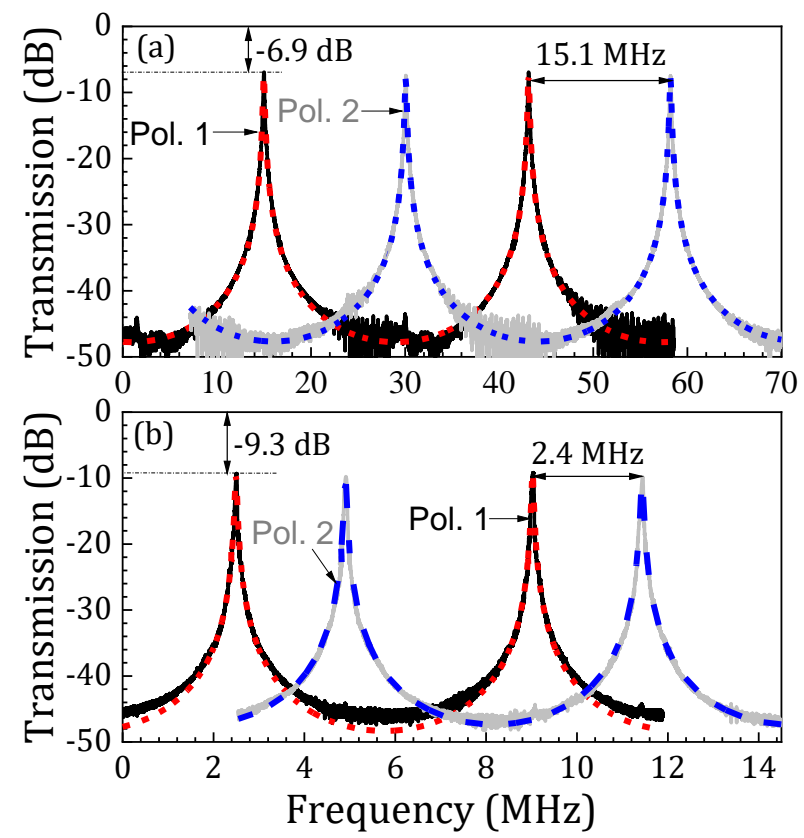

Fig. 6. Measured optical transmission spectra along the two principal axes of birefringence (Pol. 1, black and Pol. 2, grey solid) of (a) $5 \mathrm{~m}$ and (b) $23 \mathrm{~m} \mathrm{HCF}$ FPs. The insertion loss and polarization-induced spectral splitting are also shown. The red and blue dashed lines are data fitted by the transmission function of FP.
Due to the birefringence of the HCF (caused by the fabricated HCF inner structure not being perfectly symmetric and by HCF bending/coiling [21]), transmission peaks occur at two different positions within one FSR period when varying the polarization state of the launched light (via a polarization controller, Fig. 5). It is worth mentioning that this phenomenon is also observed with SMF-FPs [22] in which a very small residual fiber birefringence causes this splitting. The transmitted spectra for the two polarization eigenstates of two HCF-FPs are displayed in Fig. 6. The polarization peak spectral splitting was 15.1 and $2.42 \mathrm{MHz}$ for the 5 and $23 \mathrm{~m}$ HCF-FPs, respectively. If the two peaks for the $5 \mathrm{~m}$ HCF-FP were within the same FP resonance order (which we have not confirmed yet), HCF phase birefringence would have been $8 \times 10^{-8}$ with corresponding beat length of $20 \mathrm{~m}$.

We measured the FP's insertion loss to be $6.9 \mathrm{~dB}$ for the $5 \mathrm{~m} \mathrm{HCF}-$ FP, Fig. 6, with the fitted finesse of 166 and 154 for the two polarization eigenstates, respectively. For the $23 \mathrm{~m}$ HCF-FP, the FP insertion loss was $9.3 \mathrm{~dB}$ and the fitted finesse was 139 and 130 (corresponding to 3-dB transmission peak width of 47 and $50 \mathrm{kHz}$ ), respectively. The obtained finesses are consistent with the RF method. Based on our theoretical analysis using Eqs. (1,2), the FP insertion loss is mainly caused by the mirror loss ( $\sim 0.5 \%$ for the mirrors used). Replacing the current mirrors with improved mirrors with a loss of $0.1 \%$ would reduce the FPs insertion loss by $\sim 3 \mathrm{~dB}$. Furthermore, by improving the gluing technique (i.e., no degradation of finesse during the gluing process), we could further improve the FP insertion loss by $\sim 2 \mathrm{~dB}$ for the two HCF-FPs.

\section{Characterization of the thermal response}

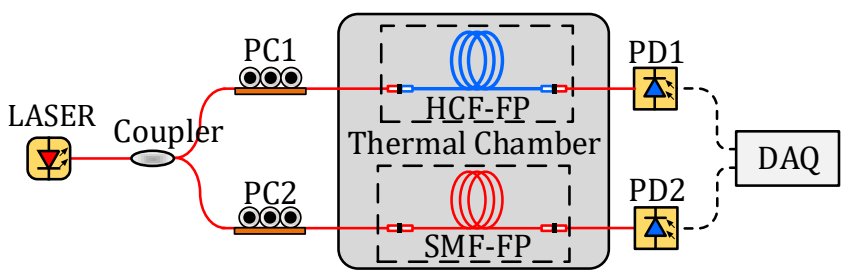

Fig. 7. Set-up for the thermal response measurement. DAQ: Data Acquisition System.

Thermal response here refers to how the transmission peaks frequency shifts with temperature. We characterized the thermal response of our $5 \mathrm{~m}$ HCF-FP using the set-up shown in Fig. 7. For comparison purposes we placed the $5 \mathrm{~m}$ HCF-FP together with a 3.6 $\mathrm{m}$ SMF-FP (which has the same optical length $n L$ as the $5 \mathrm{~m}$ HCF-FP) into the same thermal chamber. The light source (NP Photonics used earlier, buthere operated at fixed wavelength) was split and launched simultaneously into both FPs. PCs were used at both interferometer inputs to align the input polarization to one of the two FPs' eigenstates. The output power of both FPs was monitored using two photodiodes.

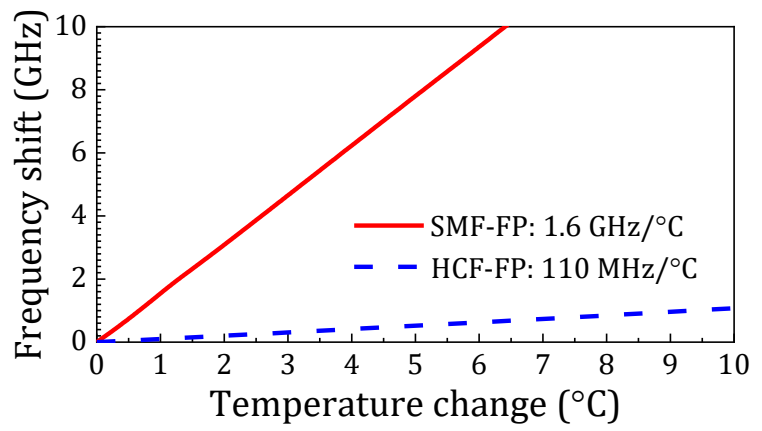

Fig. 8. Frequency shift of a selected transmission peak in the HCF-FP and a SMF-FP (of equivalent optical length) as the temperature is increased. 
From the results shown in Fig. 8 we see that the transmission peak of the HCF-FP moved at a rate of $110 \mathrm{MHz} /{ }^{\circ} \mathrm{C}$ while in the SMF-FP it moved by $1.6 \mathrm{GHz} /{ }^{\circ} \mathrm{C}$. Thus, the temperature sensitivity of HCF-FP is 14.5 times lower than that of SMF-FP, which matches well with the theoretical prediction. This is also the first demonstration of such a low sensitivity using an antiresonant HCF.

\section{CONCLUSION}

In conclusion, we fabricated 5-m and 23-m long HCF-FPs. Our fully assembled permanent-connection based FPs have finesse $>120$ over the entire $\mathrm{C}$ band, which represents the first demonstration of long length ( $>1 \mathrm{~m}$ ) HCF-FPs with such a high finesse. This was enabled by the combination of the latestgeneration of low-loss (sub-1dB/km) HCFs and the recently developed technique for permanent, low loss, fusion-splice-less sealed HCF interconnection with standard optical fibers. We achieved a FP transmission peak width as narrow as $47 \mathrm{kHz}$ (with an equivalent time delay of $3.2 \mathrm{~km}$ ). This is only a factor of three wider than for state-of-the-art bulk FP cavities that are typically $10 \mathrm{~cm}$ long and have a finesse in excess of $10^{5}$. We also showed HCF-FPs to have 14.5 times lower thermal sensitivity than SMF-FPs, which is due to the HCF's low thermal phase sensitivity. Our work represents the first demonstration of this property for antiresonant type HCF. The performance we demonstrate here, together with the expected high nonlinear threshold [9], will be of interest in many applications in which SMF-FPs cannot be used and free-space FPs are impractical.

\section{ACKNOWLEDGMENT}

The data in this paper is accessible through the University of Southampton research repository (DOI: 10.5258/SOTON/XXXX).

\section{REFERENCES}

[1] A. V. V. Nampoothiri et al., "Hollow-core Optical Fiber Gas Lasers (HOFGLAS): a review [Invited]," Opt. Mater. Express, vol. 2, no. 7, pp. 948-961, 2012.

[2] A. V. V. Nampoothiri, B. Debord, M. Alharbi, F. Gérôme, F. Benabid, and W. Rudolph, "CW hollowcore optically pumped $\mathrm{I}_{2}$ fiber gas laser," Opt. Lett., vol. 40, no. 4, pp. 605-608, Feb. 2015.

[3] M. Xu, F. Yu, M. R. Hassan, and J. C. Knight, "Continuous-Wave Mid-Infrared Gas Fiber Lasers," IEEE J. Sel. Top. Quantum Electron., vol. 24, no. 3, pp. $1-8,2018$.

[4] S. Häfner et al., " $8 \times 10^{-17}$ fractional laser frequency instability with a long room-temperature cavity," Opt. Lett., vol. 40, no. 9, pp. 2112-2115, May 2015.

[5] T. Kessler et al., "A sub-40-mHz-linewidth laser based on a silicon single-crystal optical cavity," Nat. Photonics, vol. 6, no. 10, pp. 687-692, Oct. 2012.

[6] G. Gagliardi, M. Salza, S. Avino, P. Ferraro, and P. De Natale, "Probing the Ultimate Limit of Fiber-Optic Strain Sensing," Science., vol. 330, no. 6007, pp. 10811084, Nov. 2010.

[7] J. Chen, Q. Liu, X. Fan, and Z. He, "Ultrahigh resolution optical fiber strain sensor using dual PoundDrever-Hall feedback loops," Opt. Lett., vol. 41, no. 5, pp. 1066-1069, 2016.

[8] N. M. R. Hoque and L. Duan, "Ultrahigh-Resolution Fiber-Optic Sensing Using a High-Finesse, MeterLong Fiber Fabry-Perot Resonator," Art. no. arXiv:1908.08595 [physics.ins-det], Aug. 2019.

[9] M. B. Gray, J. H. Chow, K. McKenzie, and D. E. McClelland, "Using a passive fiber ring cavity to generate shot-noise-limited laser light for low-power quantum optics applications," IEEE Photonics Technol. Lett., vol. 19, no. 14, pp. 1063-1065, 2007.

[10] R. Slavík et al., "Ultralow thermal sensitivity of phase and propagation delay in hollow core optical fibers," Sci. Rep., vol. 5, no. 1, Art. no. 15447, Dec. 2015.

[11] M. Tateda, S. Tanaka, and Y. Sugawara, "Thermal characteristics of phase shift in jacketed optical fibers," Appl. Opt., vol. 19, no. 5, pp. 770-773, 1980.

[12] S. Meiselman and G. A. Cranch, "Optical phase response to temperature in a hollow-core photonic crystal fiber," Opt. Express, vol. 25, no. 22, pp. 2758127594, Oct. 2017.

[13] J. Flannery, R. Al Maruf, T. Yoon, and M. Bajcsy, "Fabry-Pérot Cavity Formed with Dielectric Metasurfaces in a Hollow-Core Fiber," ACS Photonics, vol. 5, no. 2, pp. 337-341, Feb. 2018.

[14] Y. Tan et al., "Hollow-Core Fiber-Based High Finesse Resonating Cavity for High Sensitivity Gas Detection," J. Light. Technol., vol. 35, no. 14, pp. 2887-2893, 2017. R. Amezcua-Correa, F. Gèrôme, S. G. Leon-Saval, N. G. R. Broderick, T. A. Birks, and J. C. Knight, "Control of surface modes in low loss hollow-core photonic bandgap fibers," Opt. Express, vol. 16, no. 2, pp. 11421149, Jan. 2008.

[16] S. Gao et al., "Hollow-core conjoined-tube negativecurvature fiber with ultralow loss," Nat. Commun., vol. 9, no. 1, Art. no. 2828, 2018.

[17] T. D. Bradley et al., "Record Low-Loss $1.3 \mathrm{~dB} / \mathrm{km}$ Data Transmitting Antiresonant Hollow Core Fiber," in 2018 European Conference on Optical Communication (ECOC), 2018, no. 1, pp. 1-3.

[18] T. D. Bradley et al., "Antiresonant Hollow Core Fiber with $0.65 \mathrm{~dB} / \mathrm{km}$ Attenuation in the $\mathrm{C}$ and $\mathrm{L}$ Telecommunication Bands," in 2019 European Conference on Optical Communication (ECOC), 2019, Art. no. PDP3.1.

[19] M. Komanec et al., "Low-Loss and Low-BackReflection Hollow-Core to Standard Fiber Interconnection," IEEE Photonics Technol. Lett., vol. 31, no. 10, pp. 723-726, 2019.

[20] H. Tsuchida, "Characterization of optical resonators with an incoherent light," Opt. Express, vol. 20, no. 28, pp. 29347-29352, Dec. 2012.

[21] C. Rohrer et al, "Phase shift introduced degradation of polarization caused by bends in inhibited-coupling guiding hollow-core fibers, "IEEE Photonics Technol. Lett., vol. 31, no. 16, pp. 1362-1365, 2019.

[22] T. Yoshino, K. Kurosawa, K. Itoh, and T. Ose, "FiberOptic Fabry-Perot Interferometer and its Sensor Applications," IEEE Trans. Microw. Theory Tech., vol. 
30, no. 10, pp. 1612-1621, Oct. 1982. 